\title{
Magnetic anisotropy of $\mathrm{Cr}$ (III) ions in polymeric oxalate complexes as revealed by HF-ESR spectroscopy
}

\author{
Dijana Žilić • Lidija Androš • Yulia \\ Krupskaya · Vladislav Kataev · Bernd \\ Büchner
}

Received: date / Accepted: date

\begin{abstract}
Two new $\mathrm{Cr}(\mathrm{III})$ paramagnetic polymeric complexes:
$\left.\left\{\left[\mathrm{CaCr}_{2} \text { (phen }\right)_{2}\left(\mathrm{C}_{2} \mathrm{O}_{4}\right)_{4}\right] \cdot 0.33 \mathrm{H}_{2} \mathrm{O}\right\}_{n}$ (CrPhen) and

$\left\{\left[\mathrm{CaCr}_{2}(\mathrm{bpy})_{2}\left(\mathrm{C}_{2} \mathrm{O}_{4}\right)_{4}\right] \cdot 0.83 \mathrm{H}_{2} \mathrm{O}\right\}_{n}(\mathrm{CrBpy})$, where phen $=1,10$-phenanthroline and bpy $=2,2^{\prime}$-bipyridine, have been studied by high field-high frequency electron spin resonance (HF-ESR) spectroscopy. From the oriented powder spectra, recorded in the frequency range $83-332 \mathrm{GHz}$ in magnetic fields up to $16 \mathrm{~T}$, the spin-Hamiltonian parameters, $g, D$ and $E$, have been obtained. Both chromium complexes have a small negative (easy axis) magnetic anisotropy $|D|<1 \mathrm{~K}$. CrPhen complex is biaxial, i.e. besides axial $D$, there is also significant rhombic zero-field splitting (ZFS) parameter, E. Contrary to CrPhen, CrBpy complex is uniaxial, but described by two sets of axial ZFS parameters. Despite very similar crystallographic structures of both complexes, HF-ESR spectroscopy resolved the existence of two magnetically inequivalent $\mathrm{Cr}$ (III)

D. Žilić

Institute for Solid State Research, IFW Dresden, Helmholtzstrasse 20, D-01069 Dresden, Germany

E-mail: dzilic@irb.hr

Present address: Ruđer Bošković Institute, Bijenička cesta 54, 10000 Zagreb, Croatia

L. Androš

Ruđer Bošković Institute, Bijenička cesta 54, 10000 Zagreb, Croatia

Y. Krupskaya

Institute for Solid State Research, IFW Dresden, Helmholtzstrasse 20, D-01069 Dresden, Germany

Present address: Department of Condensed Matter Physics, Ecole de Physique 24, quai Ernest-Ansermet, CH-1205 Geneva, Switzerland

V. Kataev

Institute for Solid State Research, IFW Dresden, Helmholtzstrasse 20, D-01069 Dresden, Germany

B. Büchner

Institute for Solid State Research, IFW Dresden, Helmholtzstrasse 20, D-01069 Dresden, Germany and Institute for Solid State Physics, Technical University Dresden, D-01062 Dresden, Germany
\end{abstract}


ions in CrBpy complex. The results fully support the earlier X-ray, SQUID magnetization and powder X-band ESR studies.

Keywords $\mathrm{Cr}(\mathrm{III}) \cdot$ metal-organic complex $\cdot$ EPR $\cdot$ ESR $\cdot$ high field $\cdot$ high frequency $\cdot$ magnetic anisotropy $\cdot$ zero-field splitting

\section{Introduction}

Molecular magnets are being investigated as a part of the search for multifunctional materials as well as with respect to the fundamental understanding of magnetism of molecular materials.[1,2] Besides a development in chemical synthesis, a progress in the molecular magnetism is achieved due to the progress in experimental techniques in physics. Electron spin resonance (ESR) spectroscopy is a valuable method in solid state physics because it can provide insights into local properties of paramagnetic centers and microscopic picture of interactions in the investigated systems. However, commercial ESR spectrometers operational at a few selected frequencies below $\sim 100 \mathrm{GHz}$ do not always meet necessary requirements for investigation of transition metal complexes with spin $S>1 / 2$, in particular regarding the detection of very broad signals as well yet insufficient spectral resolution. Static susceptibility measurements can give only approximate values, particularly inaccurate for the complexes with small magnetic anisotropy of transition metal ion. In order to obtain the spin-Hamiltonian parameters: g-tensor, D-tensor (zero-field splitting) and $\mathbf{A}$-tensor (hyperfine splitting) for the complexes with spin $S>1 / 2$, it is necessary to employ ESR spectrometers that operate in high magnetic fields and at high (sub-THz) frequencies (high field-high frequency ESR, HFESR). This method was already successfully applied for determination of a large magnetic anisotropy in molecular magnets. [3-5]

Among a large number of new compounds, oxalate complexes of transition metals have drawn considerable attention in the field of molecular magnetism due to high efficiency of oxalate bridge $\mathrm{C}_{2} \mathrm{O}_{4}{ }^{2-}$ in transmitting the exchange interaction between metal ions.[6] In the preparation of homo- and heteropolynuclear compounds with interesting magnetic properties, chromium(III) mononuclear anions, such as $\left[\mathrm{Cr}(N \text {-ligand })\left(\mathrm{C}_{2} \mathrm{O}_{4}\right)_{2}\right]^{-}(N$-ligand $=$ phen/bpy, where phen $=1,10$-phenanthroline and bpy $=2,2^{\prime}$-bipyridine), are frequently used as a starting reactants.[7] Here, two new heterometallic (chromium and calcium) polymeric oxalate complexes: $\left\{\left[\mathrm{CaCr}_{2}(\text { phen })_{2}\left(\mathrm{C}_{2} \mathrm{O}_{4}\right)_{4}\right] \cdot 0.33 \mathrm{H}_{2} \mathrm{O}\right\}_{n}$ (CrPhen) and $\left\{\left[\mathrm{CaCr}_{2}(\text { bpy })_{2}\left(\mathrm{C}_{2} \mathrm{O}_{4}\right)_{4}\right] \cdot 0.83 \mathrm{H}_{2} \mathrm{O}\right\}_{n}$ (CrBpy) are studied. The crystal structures of CrPhen and CrBpy complexes are reported in Ref. [8] and Ref. [9], respectively. Molecular geometry of the metal atoms is almost identical in both complexes. In spite of analogous chemical composition of CrPhen and CrBpy complexes, they are not isostructural. However, structural motifs in both complexes are composed of mononuclear $\left[\mathrm{Cr}(N \text {-ligand })\left(\mathrm{C}_{2} \mathrm{O}_{4}\right)_{2}\right]^{-}$anions that are bridging calcium ions, and altogether form infinite, neutral heterobimetallic double zigzag chains.[9,8] Magnetic susceptibility study as well as 
X-band ESR spectroscopy showed that both polymeric complexes are paramagnetic i.e. no exchange interaction between chromium spins $S=3 / 2$ was detected.[8] At low temperatures $(T<20 \mathrm{~K})$ the susceptibility deviates from the Curie law, due to existence of the small magnetic anisotropy of $\mathrm{Cr}$ (III) ion.[8] In this work, detailed HF-ESR investigation of the anisotropy and determination of spin-Hamiltonian parameters of CrPhen and CrBpy complexes are presented. The results are interpreted in the light of the previous crystallographic and magnetic (SQUID and X-band ESR) studies.

\section{Experimental}

The HF-ESR experiments were performed with a home-made spectrometer based on a Millimeterwave Vector Network Analyzer (MVNA) from AB Millimetre, Paris, for generation of millimeter- and submillimeter microwaves and phase locked detection of a signal. The spectrometer uses a $16 \mathrm{~T}$ superconducting magnetocryostat from Oxford Instruments Ltd. For details see Ref. [10].

\section{Results}

\subsection{HF-ESR spectra}

The HF-ESR spectroscopy was performed on the powder samples of CrPhen and CrBpy complexes (polycrystalline sample was slightly ground into powder). During the measurements, small grains were self-oriented in a high magnetic field, revealed an existence of the "easy" axis of magnetic anisotropy in the complexes. The HF-ESR spectra were recorded in the frequency range $83-332 \mathrm{GHz}$, from $T=4 \mathrm{~K}$ up to $150 \mathrm{~K}$. Fig. 1 and Fig. 2 show the typical ESR spectra at four applied frequencies: 83, 166, 249 and $332 \mathrm{GHz}$.

The spectrum of CrPhen complex exhibits three separated ESR lines. Besides these main lines, at frequency $83 \mathrm{GHz}$, a weak line could be also detected at $\approx 0.7 \mathrm{~T}$. The frequency, $\nu$, vs. resonance field, $H_{\text {res }}$, dependencies i.e. three parallel resonance branches (marked with red, blue and green color) are presented, together with the corresponding spectra, in Fig. 1. From the arithmetic mean of the slopes of the resonance branches, $g$-factor could be obtained as $g=\left(h / \mu_{B}\right) \partial \nu / \partial H_{\text {res }}=1.98$. [11] Here, $h$ is the Planck constant and $\mu_{B}$ is the Bohr magneton. The left resonance branch (red color) has the $y$-intercept $\Delta=31 \mathrm{GHz} \approx 1.5 \mathrm{~K}$, that corresponds to a first estimate of the magnetic anisotropy gap, i.e. the magnitude of the zero-field splitting (ZFS) of the spin levels.

In contrast to the ESR spectrum of CrPhen that comprises three lines, the spectrum of CrBpy complex exhibits six lines, in the form of three doublets, as could be seen in Fig. 2. From the six resonance branches, two set of $g$ and $\Delta$-parameters, with similar mutual values, were obtained: $g_{1}=1.98$, 


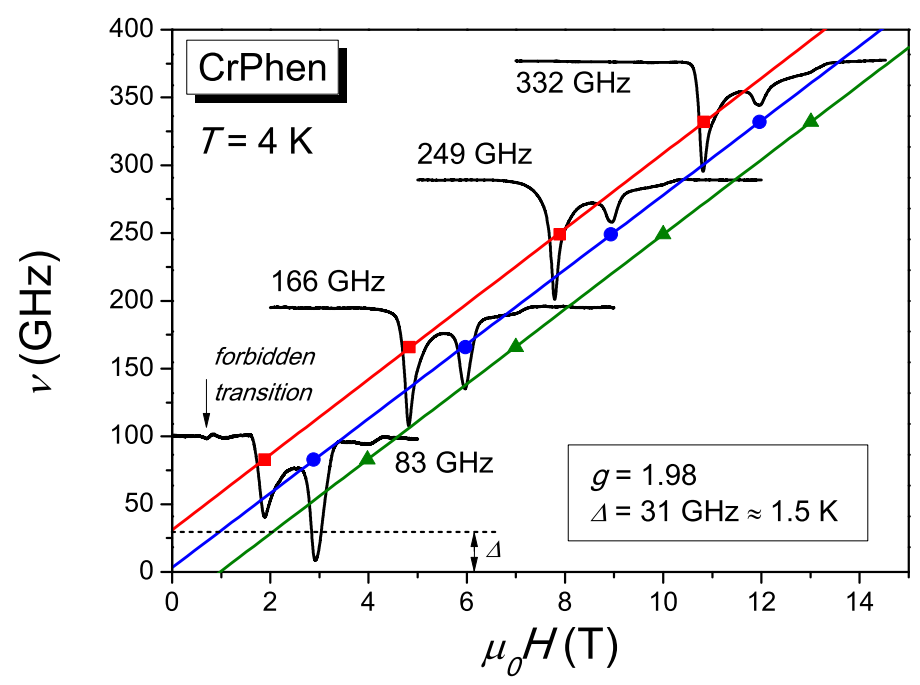

Fig. 1 The frequency dependence of the ESR spectrum of the oriented powder of CrPhen complex at $T=4 \mathrm{~K}$.

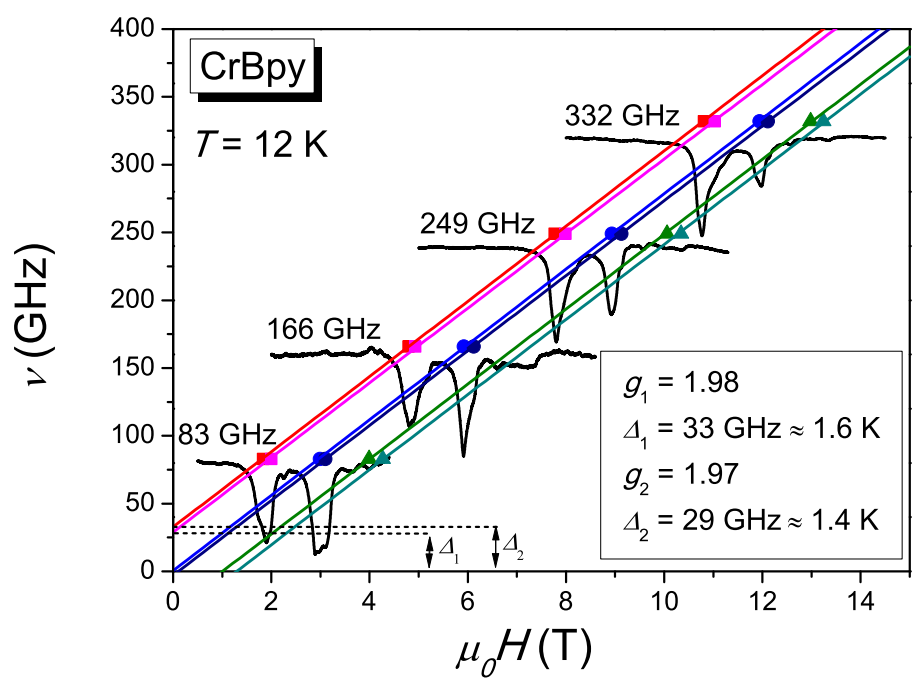

Fig. 2 The frequency dependence of the ESR spectrum of the oriented powder of CrBpy complex at $T=12 \mathrm{~K}$. 

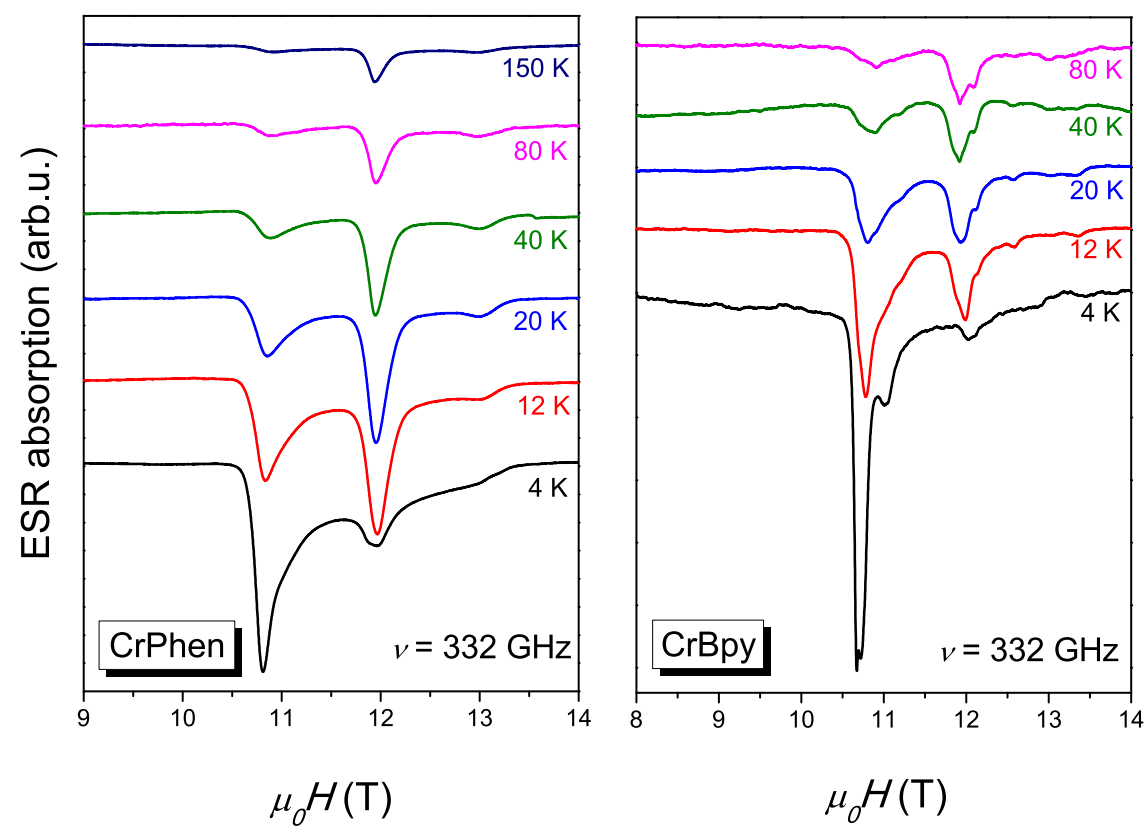

Fig. 3 The temperature dependence of the ESR spectrum of the oriented powder of CrPhen and CrBpy complexes at $\nu=332 \mathrm{GHz}$.

$\Delta_{1}=33 \mathrm{GHz} \approx 1.6 \mathrm{~K}$ and $g_{2}=1.97, \Delta_{2}=29 \mathrm{GHz} \approx 1.4 \mathrm{~K}$.

The temperature dependencies of the ESR spectra of CrPhen and CrBpy complexes, recorded at $\nu=332 \mathrm{GHz}$, are shown in Fig. 3. A transfer of the spectral weight to lower magnetic field at low temperatures revealed a negative axial magnetic anisotropy of the molecule (parameter $D<0$ ) in both complexes[11, 4], in accord with the self-orientation of the particles during measurements in a high magnetic field and the presence of an "easy" axis of anisotropy.

\subsection{Structural considerations}

Fig. 4 shows positions of $\left[\mathrm{Cr}(\mathrm{L})\left(\mathrm{C}_{2} \mathrm{O}_{4}\right)_{2}\right]^{-}$anions $(\mathrm{L}=$ phen, bpy) in one unit cell of CrPhen and CrBpy complexes, as a consequence of the symmetry operations in the $\mathrm{P} 2_{1} / \mathrm{n}$ and $\mathrm{P} \overline{1}$ space group, respectively. In both compounds there are two symmetry independent $\mathrm{Cr}$ atoms (Cr1 and $\mathrm{Cr} 2)$.

The $\left[\mathrm{Cr}(\mathrm{L})\left(\mathrm{C}_{2} \mathrm{O}_{4}\right)_{2}\right]^{-}$anion has a local two-fold rotation axis. Detailed description of calculating a position of the local rotation axis in paramagnetic chromium complex could be found in Ref. [13]. Here, the local two-fold rotation axes of $\left[\mathrm{Cr}(\mathrm{L})\left(\mathrm{C}_{2} \mathrm{O}_{4}\right)_{2}\right]^{-}$anions of symmetry independent $\mathrm{Cr}$ atoms are mutually inclined by $0.77^{\circ}$ in $\mathrm{CrBpy}$ while in CrPhen complex the inclination 


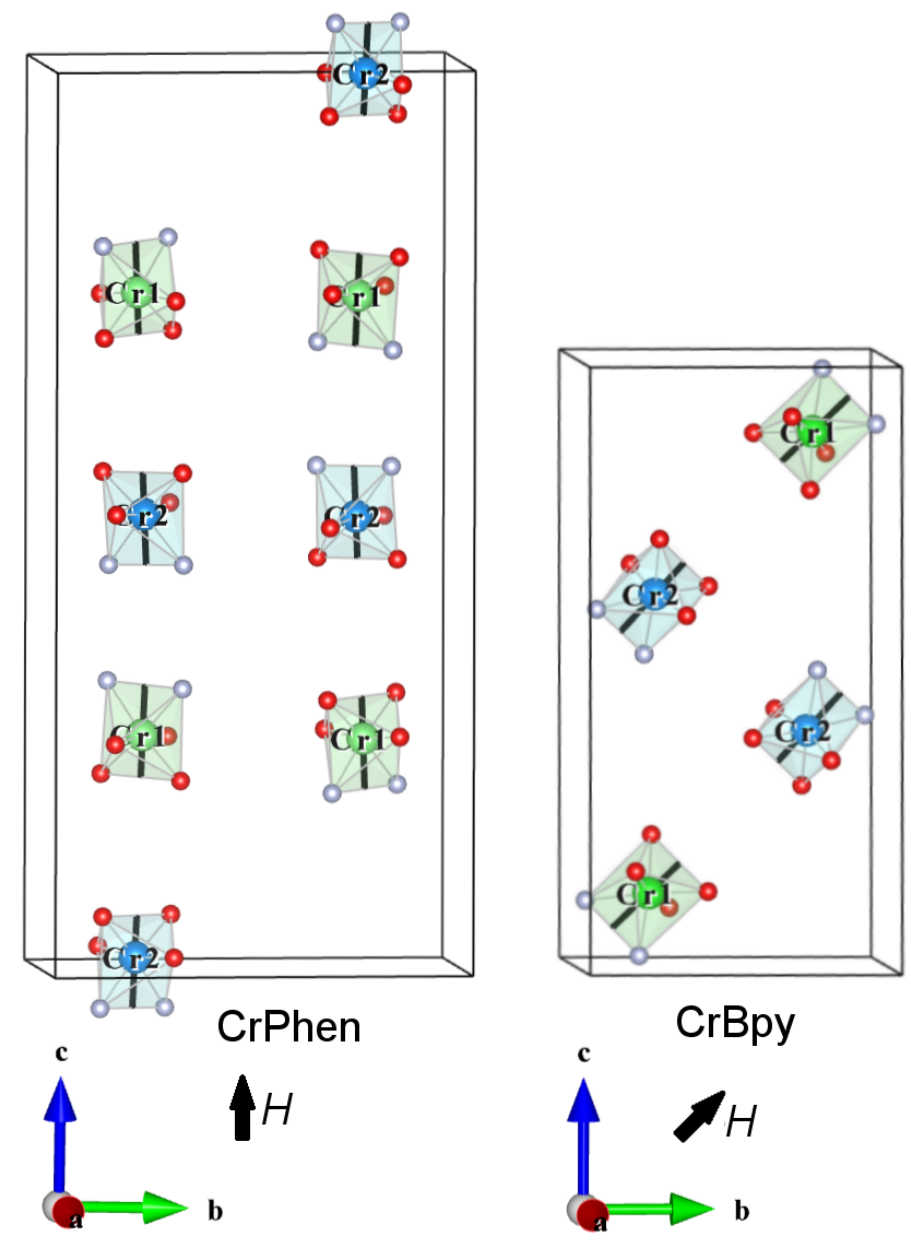

Fig. 4 Crystal packing of $\left[\mathrm{Cr}(\mathrm{L})\left(\mathrm{C}_{2} \mathrm{O}_{4}\right)_{2}\right]^{-}$anions ( $\mathrm{L}=$ phen, bpy) in the unit cell of CrPhen and CrBpy complexes. The local, approximate two-fold rotation axis (black rod) passes through the $\mathrm{Cr}$ atom and through the middle of the edges of octahedron, which connect two nitrogen (blue) atoms and two oxygen (red) atoms opposite to nitrogen atoms. For clarity, only atoms that form coordination polyhedra are depicted. Molecular graphics were prepared using VESTA.[12] $H$ indicates the direction of the external magnetic field that orientates small grains along the easy axes.

between symmetry independent octahedra is almost undetectable. Symmetry related $\mathrm{Cr}$ atoms in both compounds have identical orientation in space because they are related through the center of inversion. Since the difference in the directions of local two-fold rotation axes is almost negligible, it can be concluded that magnetic anisotropy axes of all chromium octahedra in the crystal packing of CrPhen and CrBpy complexes are approximately collinear. This 
is an agreement with the experimental observation that the small crystalline particles oriented in strong external magnetic fields.

\subsection{Analysis}

The analysis of the ESR spectra can be performed assuming the following spin Hamiltonian for the paramagnetic $\mathrm{Cr}$ (III) ion:

$$
\mathbf{H}=\mu_{B} \mathbf{B} \cdot \mathbf{g} \cdot \mathbf{S}+\mathbf{S} \cdot \mathbf{D} \cdot \mathbf{S} .
$$

Here $\mathbf{g}$ is the $\mathbf{g}$ tensor, $\mathbf{D}$ is the ZFS tensor, $\mathbf{B}$ is the magnetic field and $\mathbf{S}$ is the spin operator of $\mathrm{Cr}$ (III) ion. The first term represents the Zeeman interaction of the chromium spin $S=3 / 2$ with the external magnetic field $\mathbf{B}$. The second term describes the splitting of the spin state in the absence of magnetic field, due to anisotropy of the ligand field acting on the Cr(III) ion. Eq. 1 could be written equivalently as [14]:

$$
\mathbf{H}=g \mu_{B} \mathbf{B} \cdot \mathbf{S}+D\left[S_{z}{ }^{2}-S(S+1) / 3\right]+E\left({S_{x}}^{2}-S_{y}{ }^{2}\right)
$$

where $D$ and $E$ are the axial and rhombic ZFS parameters, respectively. The $g$-factor is approximated with an isotropic value, as is usually found for $\mathrm{Cr}$ (III) ions in distorted octahedral coordination.[11,13-16]

Here, we assume the values of the $g$-factors and $\Delta$ parameters obtained from the resonance branches, shown in Figs. 1 and 2. The relation between the axial ZFS parameter, $D$, and the magnetic anisotropy gap, $\Delta$, is: $[4,5]$

$$
|D|=\Delta /\left[S^{2}-(S-1)^{2}\right] .
$$

Therefore, for $S=3 / 2$, it follows $|D|=\Delta / 2$. The sign of $D$ is negative, as is revealed from the temperature dependence of the spectra, shown in Fig. 3.

In order to get more accurate values of spin-Hamiltonian parameters in Eq. 2, it is necessary to simulate the obtained ESR spectra. As a simulation program, EasySpin toolbox for Matlab was used.[17] Due to oriented powder samples, the simulations were performed for the case of the "easy" axis of the molecules parallel to the applied magnetic field $B$. The simulated spectra of CrPhen at $\nu=249 \mathrm{GHz}$ and CrBpy at $\nu=166 \mathrm{GHz}$, both for $T=4 \mathrm{~K}$, are presented in Figs. 5 and 6, respectively.

The spin-Hamiltonian parameters obtained from the simulations are given in Table 1. For CrPhen complex, besides the axial $D$ parameter, the rhombic $|E|$ parameter is also necessary in order to simulate the spectra. The sign of $E$ has no physical meaning, except in terms of the convention of the selected coordinate axes.[18] For CrBpy complex, parameter $E$ is set to zero, but two sets of parameters $g$ and $D$ are required to simulate six observed ESR lines.

The simulated spectra reproduce satisfactorily the experimentally observed spectra, the number of observed ESR lines as well as their relative intensities. In order to assign the experimentally observed ESR peaks to the transitions between spin levels, the energy level diagrams are also presented in Figs. 5 


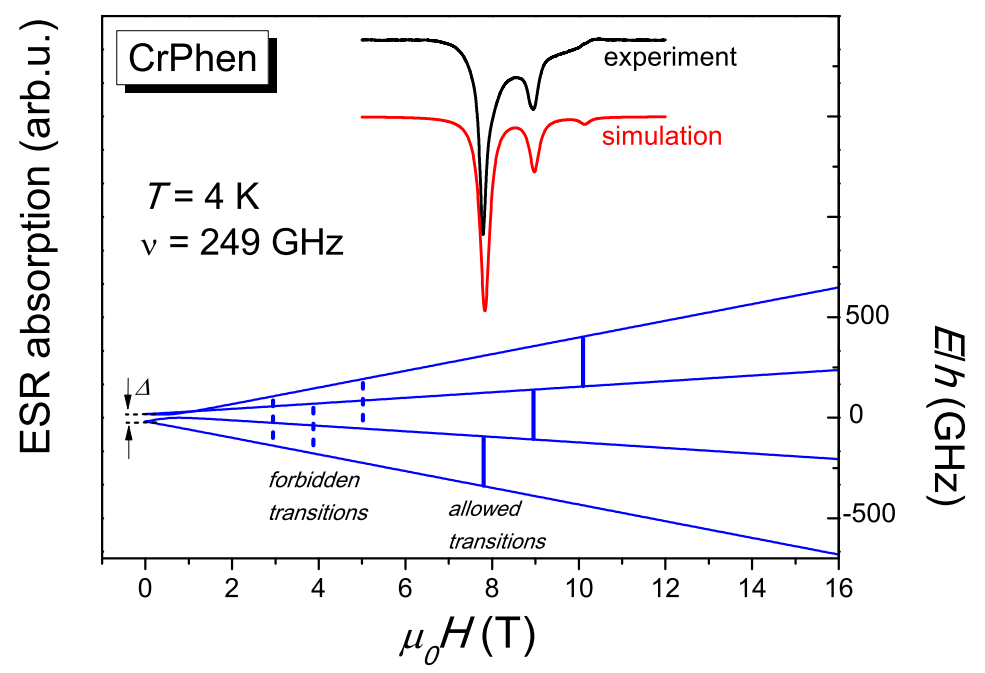

Fig. 5 Experimental and simulated ESR spectra of the oriented powder of CrPhen complex at $\nu=249 \mathrm{GHz}$ and $T=4 \mathrm{~K}$, with corresponding calculated energy levels.

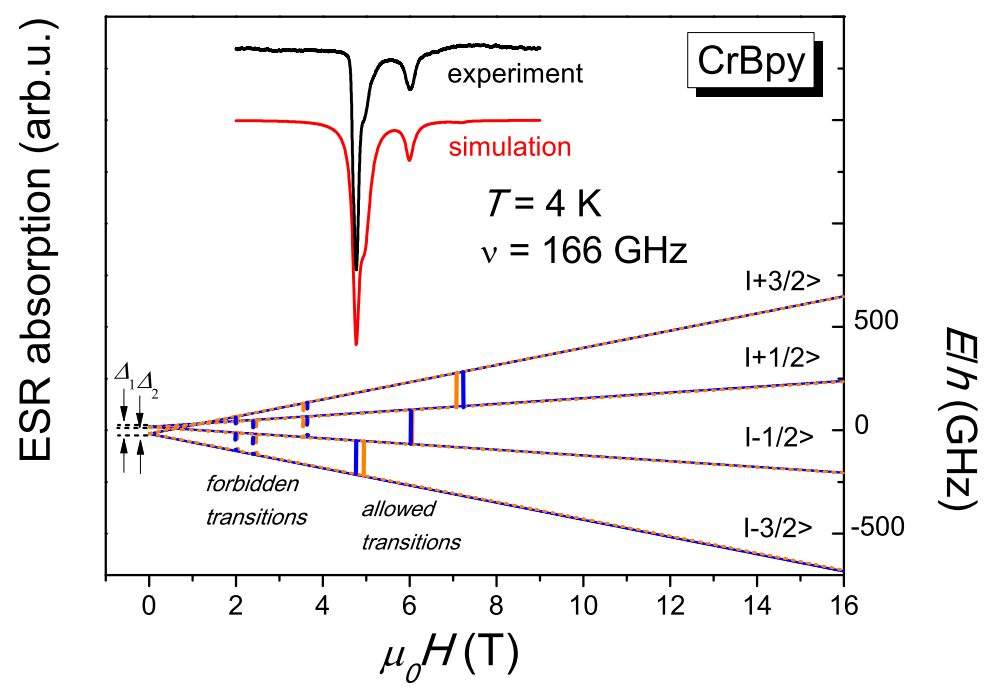

Fig. 6 Experimental and simulated ESR spectra of the oriented powder of CrBpy complex at $\nu=166 \mathrm{GHz}$ and $T=4 \mathrm{~K}$, with corresponding calculated energy levels. 
Table 1 The spin-Hamiltonian parameters $g, D$ and $E$, of CrPhen and CrBpy complexes. Two set of parameters are necessary to describe CrBpy complex.

\begin{tabular}{cccc}
\hline \multicolumn{3}{c}{ CrPhen } & \multicolumn{2}{c}{ CrBpy } \\
\hline$g$ & 1.98 & 1.98 & 1.97 \\
$D$ & $-16 \mathrm{GHz}(-0.77 \mathrm{~K})$ & $-17 \mathrm{GHz}(-0.82 \mathrm{~K})$ & $-14.5 \mathrm{GHz}(-0.70 \mathrm{~K})$ \\
$|E|$ & $5.3 \mathrm{GHz}(0.25 \mathrm{~K})$ & 0 & 0 \\
\hline
\end{tabular}

and 6. Three main lines observed in the ESR spectra of the CrPhen complex correspond to three allowed transitions $m_{s}=-3 / 2 \Longleftrightarrow m_{s}=-1 / 2, m_{s}=$ $-1 / 2 \Longleftrightarrow m_{s}=+1 / 2$ and $m_{s}=+1 / 2 \Longleftrightarrow m_{s}=+3 / 2$ (presented by solid vertical lines in Fig. 5). This is taken in the approximation that the rhombic parameter $E=0$ because, only in that case, $m_{s}$ is a good quantum number. The weak ESR line detected for $\mathrm{CrPhen}$ complex at $\approx 0.7 \mathrm{~T}$ corresponds to forbidden transitions (presented by dotted vertical lines in Fig. 5), where a selection rule $\Delta m_{s}=1$ is not valid. Similar situation can be seen in Fig. 6 for CrBpy complex. Here, two energy levels diagrams, with similar values, exist and therefore a double set of three main ESR lines was observed. The most intensive line at low temperatures is the transition $m_{s}=-3 / 2 \Longleftrightarrow m_{s}=$ $-1 / 2$, as could be seen in Fig. 3, due to the fact that the lowest energy level in the ground state is $m_{s}=-3 / 2$.

\subsection{X-band ESR spectra}

In order to check additionally the spin-Hamiltonian parameters (Table 1), obtained from the simulations HF-ESR spectra of oriented powder CrPhen and CrBpy samples, the simulations of X-band ESR spectra of the powder (non-oriented) samples were performed. The temperature dependence of the X-band ESR spectra could be found in Ref. [8]. The simulations at $T=80 \mathrm{~K}$ are presented in Fig. 7. We see a good agreement between experimentally observed and simulated powder spectra. Weak sharp peaks observed at 175, 330 and $405 \mathrm{mT}$ (marked with asterisks) originate from the X-band ESR cavity. The only line seen in the X-band spectra corresponds to $m_{s}=-1 / 2 \Longleftrightarrow m_{s}=$ $+1 / 2$ transition. Here, the role of rhombic ZFS parameter $E$ could be clearly seen; due to presence of this parameter, the line in X-band spectrum of CrPhen complex is split.

\section{Discussion}

Previously performed SQUID magnetization measurements showed paramagnetic behavior of CrPhen and CrBpy complexes, at temperatures $T>20 \mathrm{~K}$. [8] Below $20 \mathrm{~K}$, molar magnetic susceptibility $\chi(T)$ deviates from the Curie law, due to existence of ZFS.[8] The study revealed that an exchange interaction between $\mathrm{Cr}(\mathrm{III})$ ions is negligible.[8] The susceptibility fits gave $g=1.955$, $|D|=1.3 \mathrm{~K}$ and $g=1.958,|D|=2.8 \mathrm{~K}$ for CrPhen and CrBpy, respectively.[8] 


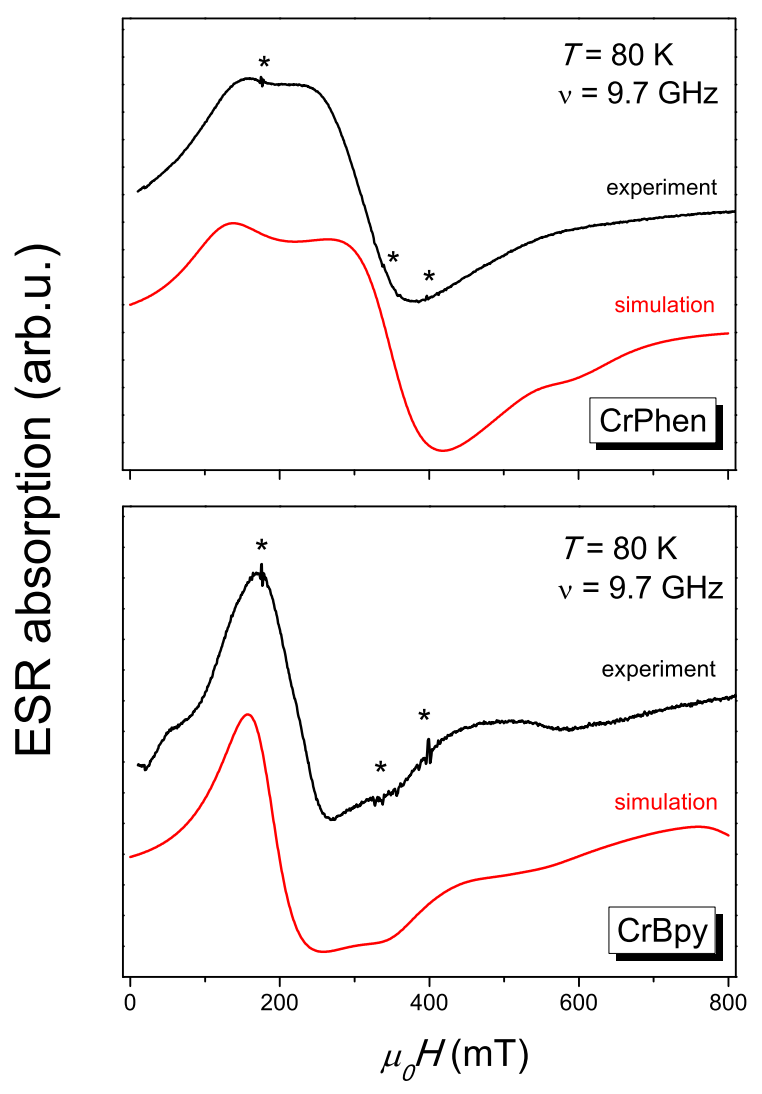

Fig. 7 Experimental and simulated ESR spectra of the powder samples of CrPhen and CrBpy complexes at $\nu=9.7 \mathrm{GHz}$ and $T=80 \mathrm{~K}$. The signals that appear at magnetic field $\mu_{0} H \approx 175,330$ and $405 \mathrm{mT}$, marked with asterisks, originate from the ESR cavity.

Comparing these values with the HF-ESR results presented in Table 1, we see only a rough agreement. In terms of energy resolution, magnetic susceptibility measurements have a sensitivity limit of $\sim 1 \mathrm{~K}$ and therefore, it is not an appropriate technique to study complexes with small values of ZFS parameter.[19] We see that in the susceptibility study the rhombic ZFS parameter, $E$, is completely neglected and two sets of spin-Hamiltonian parameters have not been resolved. Additionally, by a SQUID measurement the sign of the axial ZFS parameter $D$ has not been determined, but only its absolute value $|D|$. Besides bulk magnetization measurements, X-band ESR spectroscopy is also inadequate to study complexes of transition metal ions with spin $S>1 / 2$. From Fig. 7, one can see that only one allowed transition could be observed at X-band frequency $\left(m_{s}=-1 / 2 \Longleftrightarrow m_{s}=+1 / 2\right)$ but other two not. The exception is the single crystal $\mathrm{Cr}(\mathrm{III})$ study where it is possible, from angular 


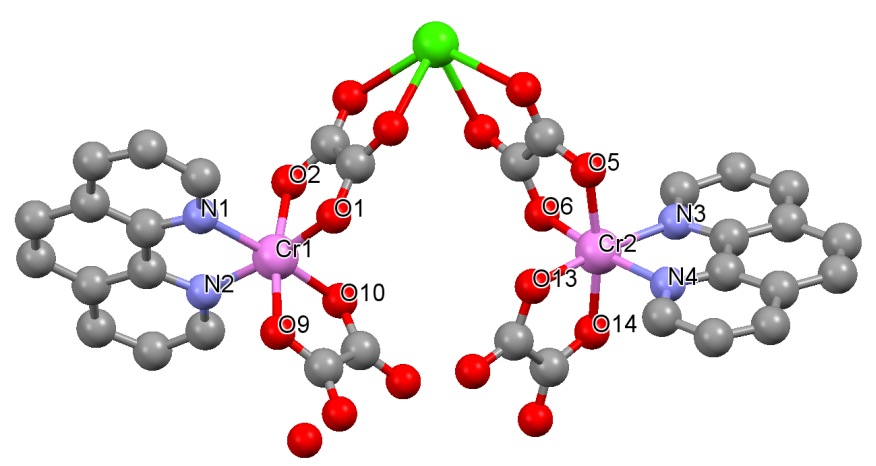

Fig. 8 The asymmetric unit of $\mathrm{CrPhen}$ complex with the atom-numbering scheme in two chromium octahedra.[8] $\mathrm{Cr}$ atoms are violet, $\mathrm{O}$ atoms are red, $\mathrm{N}$ atoms are blue, $\mathrm{C}$ atoms are grey and $\mathrm{Ca}$ atom is light green. $\mathrm{H}$ atoms have been omitted for clarity.

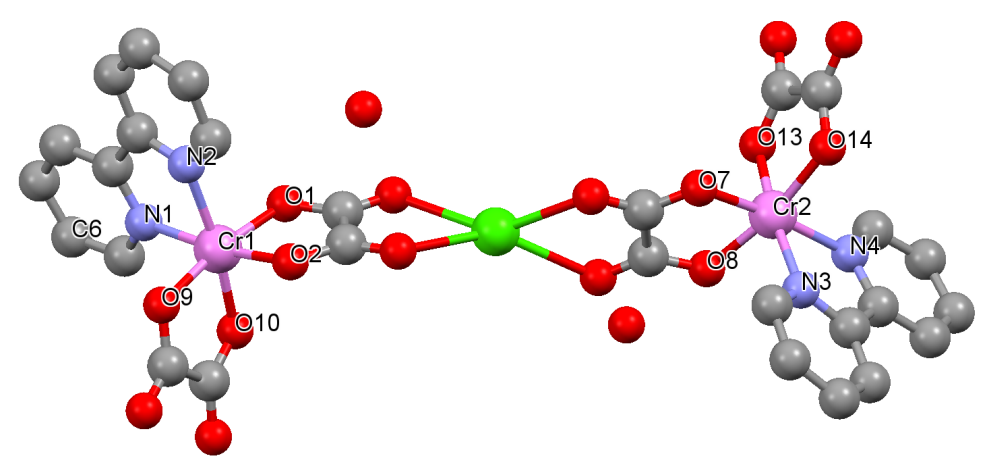

Fig. 9 The asymmetric unit of CrBpy complex with the atom-numbering scheme in two chromium octahedra.[9] $\mathrm{Cr}$ atoms are violet, $\mathrm{O}$ atoms are red, $\mathrm{N}$ atoms are blue, $\mathrm{C}$ atoms are grey and $\mathrm{Ca}$ atom is light green. $\mathrm{H}$ atoms have been omitted for clarity.

variation of the resonant field recorded in the three perpendicular planes and performing spectra simulation, to determine spin-Hamiltonian parameters, using only X-band ESR spectroscopy. $[13,16]$

Here, using HF-ESR spectroscopy, all transitions were observed and spinHamiltonian parameters were obtained. For CrBpy complex, two sets of parameters were distinguished. This indicates the existence of two magnetically inequivalent $\mathrm{Cr}$ (III) ions in the asymmetric unit of CrBpy complex. Xray diffraction showed indeed two crystallographically independent chromium atoms $\mathrm{Cr} 1$ and $\mathrm{Cr} 2$ in asymmetric unit but for both CrPhen and CrBpy compounds. $[9,8]$ All chromium atoms have a distorted octahedral coordination, involving two $\mathrm{N}$ atoms from phen/bpy ligand and four $\mathrm{O}$ atoms from two oxalate groups, as could be seen in Figs. 8 and 9. Due to the rigidity of the bidentate ligands, the donor atoms are displaced from ideal octahedral positions. 
Table 2 The values of the bond angles between opposite atoms in two chromium octahedra $(\theta$ angle) and the $\mathrm{Cr}-\mathrm{X}$ bond lengths, where $\mathrm{X}=\mathrm{N}, \mathrm{O}(x$ distance $)$ in CrPhen complex. [8] The differences between the corresponding values for $\mathrm{Cr} 1$ and $\mathrm{Cr} 2$ octahedron are also presented.

\begin{tabular}{cccccc}
\hline \hline \multicolumn{5}{c}{ CrPhen } \\
\hline \hline \multicolumn{5}{c}{ Cr1 octahedron 0 2 octahedron } \\
\hline \multicolumn{5}{c}{$\theta\left(^{\circ}\right)$} \\
\hline O2-Cr1-O9 & 170.06 & O5-Cr2-O14 & 169.91 & $|\Delta \theta|$ & 0.15 \\
O1-Cr1-N2 & 171.38 & O6-Cr2-N4 & 171.73 & $|\Delta \theta|$ & 0.35 \\
O10-Cr1-N1 & 173.99 & O13-Cr2-N3 & 172.37 & $|\Delta \theta|$ & 1.62 \\
\multicolumn{7}{c}{ sum $|\Delta \theta|$} & 2.12 \\
\hline \multicolumn{7}{c}{$x(\AA)$} \\
Cr1-N1 & 2.068 & Cr2-N3 & 2.064 & $|\Delta x|$ & 0.004 \\
Cr1-N2 & 2.072 & Cr2-N4 & 2.070 & $|\Delta x|$ & 0.002 \\
Cr1-O10 & 1.948 & Cr2-O13 & 1.950 & $|\Delta x|$ & 0.002 \\
Cr1-O1 & 1.956 & Cr2-O6 & 1.955 & $|\Delta x|$ & 0.001 \\
Cr1-O9 & 1.953 & Cr2-O14 & 1.956 & $|\Delta x|$ & 0.003 \\
Cr1-O2 & 1.974 & Cr2-O5 & 1.979 & $|\Delta x|$ & 0.004 \\
& & & sum $|\Delta x|$ & 0.016 \\
\hline \hline
\end{tabular}

Table 3 The values of the bond angles between opposite atoms in two chromium octahedra ( $\theta$ angle) and the $\mathrm{Cr}-\mathrm{X}$ bond lengths, where $\mathrm{X}=\mathrm{N}, \mathrm{O}(x$ distance $)$ in CrBpy complex. $[9]$ The differences between the corresponding values for $\mathrm{Cr} 1$ and $\mathrm{Cr} 2$ octahedron are also presented.

\begin{tabular}{|c|c|c|c|c|c|}
\hline \multicolumn{6}{|c|}{ CrBpy } \\
\hline \multicolumn{2}{|c|}{ Cr1 octahedron } & \multicolumn{2}{|c|}{ Cr2 octahedron } & \multicolumn{2}{|c|}{ Difference } \\
\hline \multicolumn{6}{|c|}{$\theta\left(^{\circ}\right)$} \\
\hline O1-Cr1-O9 & 170.95 & O8-Cr2-O14 & 169.49 & $\Delta \theta$ & 1.46 \\
\hline O10-Cr1-N2 & 171.59 & O13-Cr2-N3 & 172.21 & $\Delta \theta$ & 0.62 \\
\hline O2-Cr1-N1 & 172.30 & O7-Cr2-N4 & 172.88 & $\Delta \theta \mid$ & 0.58 \\
\hline & & & & $\operatorname{sum}|\Delta \theta|$ & 2.66 \\
\hline \multicolumn{6}{|c|}{$x(\AA)$} \\
\hline Cr1-N2 & 2.051 & Cr2-N3 & 2.055 & $\Delta x$ & 0.004 \\
\hline Cr1-N1 & 2.062 & $\mathrm{Cr} 2-\mathrm{N} 4$ & 2.065 & $\Delta x$ & 0.003 \\
\hline $\mathrm{Cr} 1-\mathrm{O} 10$ & 1.953 & $\mathrm{Cr} 2-\mathrm{O} 13$ & 1.959 & $\Delta x \mid$ & 0.006 \\
\hline $\mathrm{Cr} 1-\mathrm{O} 2$ & 1.963 & $\mathrm{Cr} 2-\mathrm{O} 7$ & 1.962 & $\Delta x \mid$ & 0.001 \\
\hline Cr1-O1 & 1.968 & $\mathrm{Cr} 2-\mathrm{O} 8$ & 1.967 & $|\Delta x|$ & 0.001 \\
\hline \multirow[t]{2}{*}{ Cr1-O9 } & 1.969 & $\mathrm{Cr} 2-\mathrm{O} 14$ & 1.977 & $|\Delta x|$ & 0.008 \\
\hline & & & & $\operatorname{sum}|\Delta x|$ & 0.023 \\
\hline
\end{tabular}

The bond angles, $\theta$, between trans positions where donor atoms ( $\mathrm{N}$ and $\mathrm{O})$ are coming from different ligands (opposite atoms in octahedra) and $\mathrm{Cr}-\mathrm{X}$ bond lengths, where $\mathrm{X}=\mathrm{N}, \mathrm{O}$ ( $x$ distance) are presented in Tables 2 and 3.

The difference in the bond angles, $|\Delta \theta|$, as well as in the bond lengths, $|\Delta x|$, between two $\mathrm{Cr}(\mathrm{III})$ ions is bigger in CrBpy complex compared to CrPhen, as could be seen from Tables 2 and 3. Therefore, two chromium octahedra are more mutually different in CrBpy compared to CrPhen complex. Two Cr(III) ions in CrPhen complex could be considered as magnetically equivalent and, therefore, one set of ESR spin-Hamiltonian parameters is sufficient to describe 
this system. In contrast, two Cr(III) ions in CrBpy complex have significantly different octahedral coordinations, which is directly reflected in their magnetic properties and leads to the existence of two sets of spin-Hamiltonian parameters.

Furthermore, from Fig. 4 it follows that the easy axis passes through the $\mathrm{Cr}$ atom and through the middle of the edges of octahedron, which connect two nitrogens and two oxygens (opposite to nitrogens). Therefore, the hard plane is approximately determined by (O2, Cr1, O9 and $\mathrm{O} 5, \mathrm{Cr} 2, \mathrm{O} 14)$ and (O1, Cr1, O9 and $\mathrm{O} 8, \mathrm{Cr} 2, \mathrm{O} 14)$ atoms for CrPhen and CrBpy complexes, respectively. Differences in these bond lengths, presented in Tables 2 and 3, are significantly bigger in CrPhen sample compared to CrBpy. The difference between Cr1-O2 and Cr1-O9 bond length is $0.021 \AA$ and between $\mathrm{Cr} 2-\mathrm{O} 5$ and $\mathrm{Cr} 2-\mathrm{O} 14$ is $0.023 \AA$ for CrPhen complex. For CrBpy complex the difference between $\mathrm{Cr} 1-$ O9 and $\mathrm{Cr} 1-\mathrm{O} 1$ is $0.001 \AA$ and between $\mathrm{Cr} 2-\mathrm{O} 14$ and $\mathrm{Cr} 2-\mathrm{O} 8$ is $0.010 \AA$. Therefore, one can conclude that structural parameters also reveal biaxial and axial magnetic structures of CrPhen and CrBpy complexes, respectively.[20, $21]$

\section{Conclusion}

HF-ESR study of two polymeric paramagnetic $\mathrm{Cr}$ (III) complexes is reported. Using frequency and temperature-dependent HF-ESR spectroscopy $(\nu=83-$ $332 \mathrm{GHz}, B=0-16 \mathrm{~T}), g$-factors, as well as $D$ - and $E$-parameters of the zero-field splitting, of chromium ions have been determined. Both complexes have negative axial ZFS parameter, $|D|<1 \mathrm{~K}$; CrPhen complex is found to be biaxial (rhombic ZFS parameter $E \neq 0$ ), while CrBpy complex is uniaxial $(E=0)$. Two $\mathrm{Cr}(\mathrm{III})$ ions in the asymmetric unit of CrPhen are approximately magnetically equivalent, while two $\mathrm{Cr}$ (III) ions in CrBpy complex have significantly different octahedral coordinations. Therefore, one and two sets of spinHamiltonian parameters are necessary to describe the magnetic anisotropy of $\mathrm{Cr}(\mathrm{III})$ ions in CrPhen and CrBpy complexes, respectively. This study shows that HF-ESR spectroscopy is a powerful method in the investigation magnetic properties of transition metal complexes.

Acknowledgements This research was supported in part by the Ministry of Science, Education and Sports of the Republic of Croatia (projects 098-0982915-2939 and 098-09829042946). The work was supported in part by the Deutsche Forschungsgemeinschaft through FOR1154 "Towards Molecular Spintronics". The work of D. Žilić at the IFW Dresden was realized through the Croatian Science Foundation (HRZZ) postdoc scholarship (project $02.03 / 164)$

\section{References}

1. D. Gatteschi, R. Sessoli, J. Villain, Molecular Nanomagnets (Oxford University Press, Oxford, UK, 2006)

2. O. Kahn, Molecular Magnetism (Wiley-VCH Inc., 1993) 
3. J. Nehrkorn, B.M. Martins, K. Holldack, S. Stoll, H. Dobbek, R. Bittl, A. Schnegg, Mol. Phys. 111, 2696 (2013)

4. Y. Krupskaya, A. Alfonsov, A. Parameswaran, V. Kataev, R. Klingeler, G. Steinfeld, N. Beyer, M. Gressenbuch, B. Kersting, B. Büchner, ChemPhysChem 11, 1961 (2010)

5. A. Das, K. Gieb, Y. Krupskaya, S. Demeshko, S. Dechert, R. Klingeler, V. Kataev, B. Büchner, P. Müller, F. Meyer, J. Am. Chem. Soc. 133, 3433 (2011)

6. M. Clemente-Leon, E. Coronado, C. Marti-Gastaldo, F.M. Romero, Chem. Soc. Rev. 40, $473(2011)$

7. G. Marinescu, M. Andruh, F. Lloret, M. Julve, Coord. Chem. Rev. 255, 161 (2011)

8. L. Androš, M. Jurić, J. Popović, D. Pajić, K. Zadro, K. Molčanov, D. Žilić, P. Planinić, Eur. J. Inorg. Chem. pp. 5703-5713 (2014)

9. L. Androš, M. Jurić, K. Molčanov, P. Planinić, Dalton Trans. 41, 14611 (2012)

10. C. Golze, A. Alfonsov, R. Klingeler, B. Büchner, V. Kataev, C. Mennerich, H.H. Klauss, M. Goiran, J.M. Broto, H. Rakoto, S. Demeshko, G. Leibeling, F. Meyer, Phys. Rev. B 73, 224403 (2006)

11. P. Chaudhuri, V. Kataev, B. Büchner, H.H. Klauss, B. Kersting, F. Meyer, Coord Chem. Rev. 253, 2261 (2009)

12. K. Momma, F. Izumi, J. Appl. Crystallogr. 44, 1272 (2011)

13. N. Novosel, D. Žilić, D. Pajić, M. Jurić, B. Perić, K. Zadro, B. Rakvin, P. Planinić, Solid State Sci. 10, 1387 (2008)

14. A. Carrington, M.A. D., Introduction to Magnetic Resonance (Harper and Row, New York, 1967)

15. S. Khanra, B. Biswas, C. Golze, B. Buchner, V. Kataev, T. Weyhermuller, P. Chaudhuri, Dalton Trans. pp. 481-487 (2007)

16. M. Jurić, P. Planinić, D. Žilić, B. Rakvin, B. Prugovečki, D. Matković-Čalogović, J. Mol. Struct. 924-926, 73 (2009)

17. S. Stoll, A. Schweiger, J. Magn. Reson. 178, 42 (2006)

18. J.A. Weil, J.R. Bolton, J.E. Wertz, Electron Paramagnetic Resonance (John Wiley and Sons, Inc., New York, 1994)

19. A. Bencini, D. Gatteschi, Electron Paramagnetic Resonance of Exchange Coupled Systems (Springer-Verlag, Berlin Heidelberg, 1990)

20. J. Telser, L.A. Pardi, J. Krzystek, L.C. Brunel, Inorg. Chem. 37, 5769 (1998)

21. J. Wang, Z. Wang, R.J. Clark, A. Ozarowski, J. van Tol, N.S. Dalal, Polyhedron 30 $3058(2011)$ 